Highlight

\title{
Catalysis on a metal surface with a graphitic cover
}

\author{
Qiang $\mathrm{Fu}^{*}$, Xinhe Bao
}

In heterogeneous catalysis, the modulation of catalysis occurring on metal catalysts can be done by changing the electronic structure of the active surface through a modification of its composition and structure of the surface or the sub-surface [1]. Surface alloying is an effective method, and the electronic structure of the metal surface can be tuned by introducing one or more metals at the surface. The introduction of another metal into the subsurface region is another way to control surface activity by the shifting of the d-band center of the metal surface. In contrast to the structural modification of the metal surface or subsurface, recent work have shown that surface activity can also be altered by placing a cover on top of the metal surface. Catalysis on a metal surface confined under a cover can present a new way to control surface reactions (Fig. 1).

When an active metal surface is coated by an inert layer, it is common knowledge that the surface active sites are blocked by the overlayer and the catalyst gets deactivated. However, the emergence of two-dimensional (2D) atomic structures such as graphene and hexagonal boron nitride ( $h-\mathrm{BN})$ [2] can change this view. These 2D atomic structures are characterized by strong in-plane bonding but weak out-of-plane interactions. In the case of a 2D atomic sheet placed on a metal surface, the 2D cover interacts with the substrate weakly through van der Waals interaction. On the other hand, many molecules can bond to the metal surface strongly by chemisorption. Therefore, the energy to move the $2 \mathrm{D}$ cover from the metal surface can be compensated by the energy decrease from molecule adsorption on the metal, and it is expected that molecules would interca-
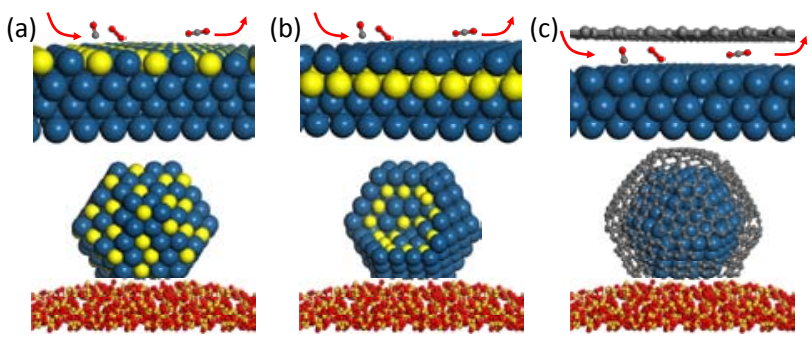

Fig. 1. Schematic illustration of typical ways to modulate metal catalysts. (a) surface alloying at the metal surface; (b) introduction of subsurface elements under the metal surface; (c) placing a graphitic cover on the metal surface. The upper row shows the model catalyst systems and the lower row shows the supported catalysts. late between the 2D cover and the metal surface. Based on this energetics consideration, the space between the top cover and metal surface can be regarded as a 2D nanoreactor in which molecules can be trapped by intercalation at the cover/metal interface and their catalytic reactions can occur there (Fig. $1(c))$.

Recent results have confirmed that many gas molecules can intercalate graphene overlayers grown on various metal surfaces. Our group first reported oxygen intercalation of the graphene overlayer on metal when exposing the graphene/ $\mathrm{Ru}(0001)$ surface to $1.6 \times 10^{-6}$ mbar $\mathrm{O}_{2}$ at $600 \mathrm{~K}$ [3]. Sutter et al. [4] have shown that the intercalated 0 species formed an ordered $\mathrm{p}(2 \times 1)-0$ adlayer structure on $\mathrm{Ru}(0001)$ grown on $\mathrm{Pt}(111)$ in the graphene. A similar oxygen intercalation process was observed with other graphene structures such as graphene/Ir, graphene/Cu, and graphene/Rh surfaces. CO intercalates under graphene islands under $10^{-6}$ torr $\mathrm{CO}$ at room temperature, while 0.01 torr $\mathrm{CO}$ is required to intercalate the full graphene layer on $\operatorname{Pt}(111)$ [5,6]. On the graphene/ $\mathrm{Ru}(0001)$ surface, CO intercalation was observed under 10 torr CO [7]. The threshold CO pressure to trigger CO intercalation increases with the interaction strength of graphene with the metal. In addition, Feng et al. [8] have shown that water efficiently splits graphene from $\mathrm{Ru}(0001)$ at a temperature as low as $90 \mathrm{~K}$, forming a $\mathrm{Gr} / \mathrm{H}_{2} \mathrm{O} / \mathrm{Ru}(0001)$ surface structure. All these results demonstrated that small molecules such as $\mathrm{O}_{2}, \mathrm{CO}$, and $\mathrm{H}_{2} \mathrm{O}$ can intercalate graphene overlayers grown on metals. Very recent results showed that the intercalation of oxygen and hydrogen occurred under BN layers grown on metals as well [9-11].

The intercalated molecules interact simultaneously with the metal and the cover, and are thus well confined in the direction vertical to the surface. It is expected that the surface chemistry of the molecules trapped there would be significantly affected. The surface chemistry of CO at the graphene/Pt(111) interface presents three new features (Fig. 2(a), (b)) [6]: (1) the C-O stretching frequency has a red shift of $14 \mathrm{~cm}^{-1}$ compared to that on the clean Pt(111) surface; (2) the CO desorption kinetics changed from first order on the clean Pt(111) surface to zero order under the graphene cover; (3) the desorption temperature of $\mathrm{CO}$ under the graphene cover was significantly 

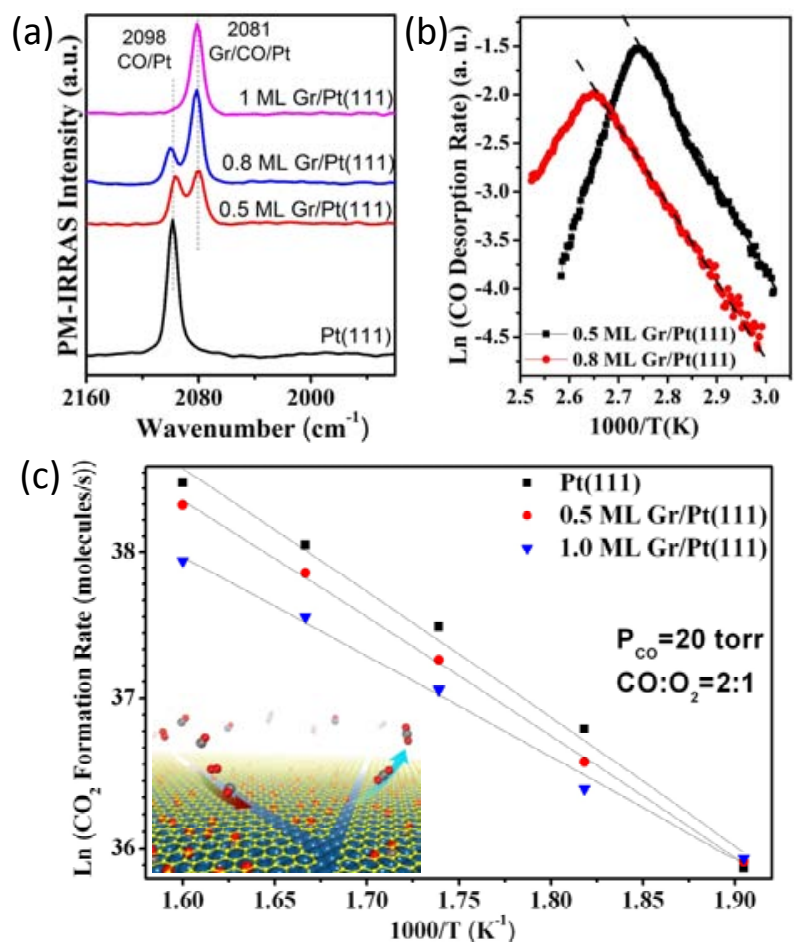

Fig. 2. Modulated surface chemistry of $\mathrm{CO}$ on Pt under a graphene cover (a) polarization-modulation infrared reflection absorption spectroscopy(PM-IRRAS) spectra of CO on $\mathrm{Pt}(111)$ and graphene/Pt(111) surfaces under 20 torr $\mathrm{CO}$ and 10 torr $\mathrm{O}_{2}$ at $300 \mathrm{~K}$. Red shift of the $\mathrm{CO}$ stretching frequency can be seen for CO under the graphene. (b) Arrhenius plots of the desorption rate of intercalated $\mathrm{CO}$ on 0.5 and 0.8 monolayer (ML) Gr/Pt(111) surfaces. The dashed line shows the linear dependence of $\mathrm{Ln}(\mathrm{CO}$ desorption rate) versus the inverse of the temperature, indicating zero order desorption kinetics. (c) Arrhenius plots of $\mathrm{CO}_{2}$ formation rate on the $\mathrm{Pt}(111)$ surface and the $\mathrm{Gr} / \mathrm{Pt}(111)$ surface in the temperature range of 525 to $625 \mathrm{~K}$ (figure taken from [6]).

lower. These results confirmed that the graphene cover exerted a strong confinement effect on the $\mathrm{CO}$ interaction with Pt. A similar rapid and abrupt $\mathrm{CO}$ desorption at a lower temperature, which is characteristic of zero order desorption kinetics, was observed on the CO-intercalated graphene/Ru(0001) surface [7]. Both experimental data [4-7,10] and density functional theory (DFT) calculations $[6,12]$ revealed a general trend that adsorption of molecules on the metal was much weakened by the graphene or h-BN cover. It is believed that the decrease in the adsorption energy is due to the energy penalty caused by the lifting of the top cover. A recent DFT work also confirmed the weakened adsorption of many molecules on metal clusters encapsulated inside one-dimensional (1D) carbon nanotube (CNT) due to the unique confined environment of the CNT channels [13]. The similar confinement effect in the 1D channel of CNTs and 2D space under a graphene cover suggested that the molecule-metal interaction can be effectively tuned by the nanosized confinement environment.

The 2D space between the top cover and the metal surface can be used as a nanoreactor where the intercalated molecules can react on the metal, dissociate on the surface, or react with each other when there are more than two kinds of intercalated molecules (Fig. 1(c)). Since the graphene cover affects the molecule-metal interaction, catalytic reactions occurring under the graphene cover would be strongly modulated by the top cover. Surface reactions such as CO oxidation $[5,6,14,15]$ and the electrolysis of water [16] have been demonstrated to occur on metal surfaces covered by graphene overlayers. Taking CO oxidation on the graphene/Pt(111) surface as an example, the catalytic reaction kinetics has been investigated and compared for the clean Pt(111) and graphene/Pt(111) surfaces. We observed that $\mathrm{CO}$ oxidation in the confined space has a lower apparent activation energy compared to the open Pt surface (Fig. 2(c)) [6]. DFT calculation revealed that the graphene overlayer decreased the bonding strength of CO with Pt such that there is a flatter potential energy surface on the reaction path and a lower reaction barrier is expected. Zhou et al. [17] reported that the graphene coating accelerated the long term oxidation of the underlying $\mathrm{Cu}$ surface under ambient atmosphere at room temperature. The promotion effect of a graphene overlayer on the surface reactions underneath has been clearly demonstrated, which contrasts sharply with the conventional view that graphitic carbon poisons a catalyst surface. This opens up a new avenue to enhance catalytic activity by coating metal catalysts with controlled graphitic covers.

These results from the model systems enable us to suggest the concept of "Catalysis under a Cover", in which the molecule-metal interaction and metal-catalyzed reactions were effectively modulated by the graphitic cover. As of now, this concept has been applied to graphene/metal and BN/metal systems. Possibly, other 2D layered structures, e.g., sulfides, graphitic oxides, and metal-organic framework (MOF) layers can perform the same function as graphene and BN. Furthermore, in order to make this concept usable in practice, the structural configuration consisting of a 2D cover on a metal surface should be constructed for supported metal catalysts. For this purpose, metal nanoparticle catalysts need to be encapsulated by a few-layer graphitic cover to form a core-shell nanostructure (Fig. 1(c)). Reactants would penetrate into the cover/metal interface and the catalytic reaction occurs in the space between the core and shell. Metal cores wrapped with a graphitic shell may have high activity/selectivity in some reactions due to the confinement effect of the top cover, and also have enhanced stability against sintering because of the protecting shell.

\section{Qiang Fu}

Dalian Institute of Chemical Physics,

Chinese Academy of Sciences

Tel: +86-411-84379253

Fax: +86-411-84694447

E-mail: qfu@dicp.ac.cn

Received 23 January 2015

Accepted 1 March 2015

Published 20 April 2015

DOI: 10.1016/S1872-2067(15)60828-2

This work was financially supported by the National Natural Science Foundation of China (21222305, 21373208, and 21033009), the National Basic Research Program of China ( 973 Program, 2011CB932704 and 2013CB834603), and the Key Research Program of the Chinese Academy of Sciences. 


\section{Graphical Abstract}

Chin. J. Catal., 2015, 36: 517-519 doi: 10.1016/S1872-2067(15)60828-2

\section{Catalysis on a metal surface with a graphitic cover}

Qiang Fu*, Xinhe Bao

Dalian Institute of Chemical Physics, Chinese Academy of Sciences

The surface reactivity of a metal can be modulated by placing a graphitic cover atop, and a concept of "Catalysis under Cover" has been suggested acting as a new route towards the modulation of surface reactions.

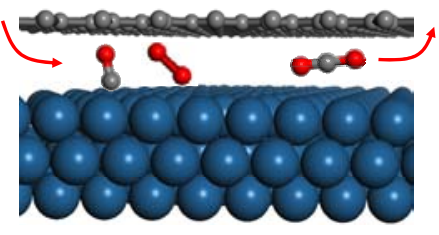

Graphene/metal model system

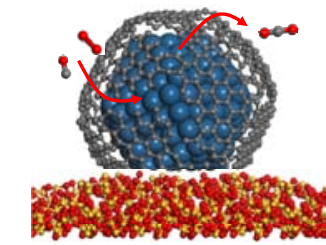

Metal@graphene nanocatalyst

\section{References}

[1] Nørskov J K, Bligaard T, Rossmeisl J, Christensen C H. Nat Chem, 2009, 1: 37

[2] Novoselov K S, Jiang D, Schedin F, Booth T J, Khotkevich V V, Morozov S V, Geim A K. Proc Natl Acad Sci USA, 2005, 102: 10451

[3] Zhang H, Fu Q, Cui Y, Tan D L, Bao X H. J Phys Chem C, 2009, 113: 8296

[4] Sutter P, Sadowski J T, Sutter E A. J Am Chem Soc, 2010, 132: 8175

[5] Mu R, Fu Q, Jin L, Yu L, Fang G Z, Tan D L, Bao X H. Angew Chem Int $E d, 2012,51: 4856$

[6] Yao Y X, Fu Q Zhang Y Y, Weng X F, Li H, Chen M S, Jin L, Dong A Y, Mu R T, Jiang P, Liu L, Bluhm H, Liu Z, Zhang S B, Bao X H. Proc Natl Acad Sci USA, 2014, 111: 17023

[7] Jin L, Fu Q, Dong A Y, Ning Y X, Wang Z J, Bluhm H, Bao X H. J Phys Chem C, 2014, 118: 12391

[8] Feng X F, Maier S, Salmeron M. J Am Chem Soc, 2012, 134: 5662

[9] Yang Y, Fu Q, Wei M M, Bluhm H, Bao X H. Nano Res, 2015, 8: 227

[10] Brugger T, Ma H F, Iannuzzi M, Berner S, Winkler A, Hutter J, Osterwalder J, Greber T. Angew Chem Int Ed, 2010, 49: 6120

[11] Kidambi P R, Blume R, Kling J, Wagner J B, Baehtz C, Weatherup R S, Schlögl R, Bayer B C, Hofmann S. Chem Mater, 2014, 26: 6380

[12] Andersen M, Hornekær L, Hammer B. Phys Rev B, 2014, 90: 155428

[13] Xiao J P, Pan X L, Guo S J, Ren P J, Bao X H. J Am Chem Soc, 2015, 137: 477

[14] Zhang Y H, Fu Q Cui Y, Mu R T, Jin L, Bao X H. Phys Chem Chem Phys, 2013, 15: 19042

[15] Grånäs E, Knudsen J, Schröder U A, Gerber T, Busse C, Arman M A, Schulte K, Andersen J N, Michely T. ACS Nano, 2012, 6: 9951

[16] Gao L B, Ren W C, Xu H L, Jin L, Wang Z X, Ma T, Ma L P, Zhang Z Y, Fu Q, Peng L M, Bao X H, Cheng H M. Nat Commun, 2012, 3: 699

[17] Zhou F, Li Z T, Shenoy G J, Li L, Liu H T. ACS Nano, 2013, 7: 6939 